of targeted response. PSAID-12 appears to be an effective composite index for retaining the response of the treatment in biological registry.

Disclosure of Interest: None declared

DOI: 10.1136/annrheumdis-2018-eular.5155

\section{AB0954 A RANDOMISED, DOUBLE-BLIND TRIAL COMPARING THE EFFICACY, SAFETY AND IMMUNOGENICITY OF MSB11022, A PROPOSED BIOSIMILAR OF ADALIMUMAB, VERSUS ADALIMUMAB ORIGINATOR IN PATIENTS WITH MODERATE-TO-SEVERE PLAQUE PSORIASIS}

J. Hercogová ${ }^{1}$, K.A. Papp ${ }^{2}$, C.J. Edwards ${ }^{3}$, V. Chyrok ${ }^{4, *}$, T. Halady ${ }^{4}$, M. Ullmann ${ }^{4}$, P. Vlachos ${ }^{5} .{ }^{1}$ Charles University, Prague, Czech Republic, ${ }^{2}$ Probity Medical Research Inc, Waterloo, ON, Canada, ${ }^{3}$ University of Southampton, Southampton, UK, ${ }^{4}$ Fresenius Kabi, Aubonne, ${ }^{5}$ Cytel, Geneva, Switzerland

Background: Adalimumab is a fully human anti-TNF mAb, indicated for the treatment of multiple inflammatory disorders. MSB11022 is a proposed adalimumab biosimilar that has shown analytical similarity [1] and bioequivalence to US licensed and EU-approved adalimumab originator, as well as comparable safety, tolerability and immunogenicity in a phase I trial [2]

Objectives: The aims of this multicentre, double-blind, parallel-group, 52-week phase III study (AURIEL-PsO, NCT02660580) were to demonstrate equivalence in efficacy (Psoriasis Area and Severity Index [PASI] 75) and to compare the safety and immunogenicity of MSB11022 vs. adalimumab originator in patients with moderate-to-severe chronic plaque psoriasis. This study was designed inline with the biosimilar regulatory framework as part of the totality of evidence to confirm similarity and rationale for extrapolation.

Methods: A total of 443 eligible patients ( 391 evaluable, including 43 with psoriatic arthritis) from 69 sites in 12 countries were randomised 1:1 and treated with MSB11022 ( $n=202)$ or adalimumab originator $(n=189)$ (80 mg subcutaneously [SC] on day $1 ; 40 \mathrm{mg} \mathrm{SC}$ every other week from weeks $2-14$ ). The primary endpoint was PASI 75 at week 16; equivalence was established if the $95 \%$ confidence interval $(\mathrm{Cl})$ for the treatment difference was within $\pm 18 \%$. Secondary endpoints included $\%$ change from baseline in PASI (equivalence confirmed if $95 \% \mathrm{Cl}$ within $\pm 15 \%$ ), Physician Global Assessment (PGA), quality of life (QoL), immunogenicity and safety. Interim results at week 16 are presented.

Results: Patient baseline characteristics were comparable between MSB11022 and adalimumab originator groups: mean age 44.8 vs. 42.4 years, male $66.8 \%$ vs. $68.3 \%$, mean PASI score 20.7 vs. 21.2 , respectively. PASI 75 scores were $89.6 \%$ for MSB11022 and $91.5 \%$ for adalimumab originator (difference $-1.9 \%[95 \% \mathrm{Cl}$ $-7.82-4.16]$ ). Mean $\%$ change from baseline in PASI was $-90.6 \%$ for MSB11022 and $-91.7 \%$ for adalimumab originator (difference $-1.0 \%[95 \% \mathrm{Cl}-1.23-2.98]$ ). PGA and QoL scores were comparable between treatment groups. The incidence of treatment-emergent adverse events (TEAEs)/serious TEAEs was $51.1 / 3.6 \%$ for MSB11022 and 53.2/2.7\% for adalimumab originator. Immunogenicity profiles of MSB11022 and adalimumab originator were also similar and consistent.

Conclusions: Week 16 results of this phase III confirmatory study demonstrated equivalent efficacy and similar safety and immunogenicity profiles for MSB11022 vs. adalimumab originator at 16 weeks in patients with moderate-to-severe chronic psoriasis

\section{REFERENCES:}

[1] Magnenat L, et al. Demonstration of physicochemical and functional similarity between the proposed biosimilar adalimumab MSB11022 and Humira ${ }^{\circledR}$. MAbs. 2017:9:127-139.

[2] Hyland E, et al. Comparison of the pharmacokinetics, safety, and immunogenicity of MSB11022, a biosimilar of adalimumab, with Humira ${ }^{\circledR}$ in healthy subjects. Br J Clin Pharmacol. 2016;82:983-93.

Disclosure of Interest: J. Hercogová Grant/research support from: Fresenius Kabi, Consultant for: Fresenius Kabi, K. Papp Grant/research support from: Fresenius Kabi, C. Edwards Grant/research support from: Fresenius Kabi, Consultant for: Fresenius Kabi, V. Chyrok Employee of: Fresenius Kabi, T. Halady Employee of: Fresenius Kabi, M. Ullmann Employee of: Fresenius Kabi, P. Vlachos Consultant for: Fresenius Kabi

DOI: 10.1136/annrheumdis-2018-eular.5146

\section{AB0955 \\ TILDRAKIZUMAB EFFICACY OVER TIME BY WEEK 28 RESPONSE LEVELS IN TWO PHASE 3 CLINICAL TRIALS IN PATIENTS WITH CHRONIC PLAQUE PSORIASIS}

A. Blauvelt ${ }^{1}$, H. Sofen ${ }^{2}$, K. Papp ${ }^{3}$, M. Gooderham ${ }^{4}$, Y. Zhao $^{5}$, S. Lowry ${ }^{5}$, A. Mendelsohn ${ }^{5}$, J. Parno ${ }^{5}$, Q. Li ${ }^{6}$, C.L. Rosa ${ }^{6}$, K. Reich . ${ }^{1}$ Oregon Medical Research Center, Portland; ${ }^{2}$ Medicine, University of Carlifornia at Los Angeles, Los Angeles, USA; ${ }^{3}$ Probity Medical Research, Waterloo; ${ }^{4}$ Skin Centre for Dermatology, Peterborough, Canada; ${ }^{5}$ Sun Pharmaceuticals, Princeton; ${ }^{6}$ Merck and Co., Inc., Kenilworth, USA; ${ }^{7}$ SClderm Research Institute and Dermatologikum, Hamburg, Germany

Background: Tildrakizumab (TIL), a high affinity, humanised, IgG1/א monoclonal antibody for IL-23p19, recently demonstrated efficacy in patients with chronic plaque psoriasis in two, phase 3 clinical trials.

Objectives: To examine efficacy from baseline to week 52 among TIL patients achieving various Psoriasis Area and Severity Index (PASI) responses at week 28.

Methods: ReSURFACE 1 (NCT01722331) and reSURFACE 2 (NCT01729754) were double-blind, randomised controlled trials in subjects with moderate-tosevere chronic plaque psoriasis ${ }^{1}$. Part 1 (0-12 weeks) was placebo controlled; Part 2 (12-28 weeks) re-randomised placebo patients to TIL; Part 3 (28-64 weeks, reSURFACE 1; 28-52 weeks, reSURFACE 2) patients with $\geq$ PASI 50 were re-randomised to continue or increase TIL dose or to placebo based on response at week 28 . In this post-hoc pooled analysis, patients on TIL $100 \mathrm{mg}$ and $200 \mathrm{mg}$ from baseline to week 52 were classified in 5 mutually exclusive groups based on their week-28 PASI response: PASI <50, PASI 50-74, PASI 7589, PASI 90-99, and PASI 100. Baseline characteristics and\% PASI improvement from baseline up to week 52 (observed data) were examined for each group. Results: This analysis included 575 (TIL $100 \mathrm{mg}$ ) and 581 (TIL $200 \mathrm{mg}$ ) patients the overall pooled Week 28 PASI 75/90/100 responses were $77 \% / 54 \% / 23 \%$ (TIL $100 \mathrm{mg}$ ) and $78 \% / 58 \% / 29 \%$ (TIL $200 \mathrm{mg}$ ). At week 28, $133(23.1 \%), 175$ (30.4\%), $137(23.8 \%), 82(14.3 \%)$, and $48(8.3 \%)$ TIL $100 \mathrm{mg}$ patients and 170 (29.3\%), 169 (29.1\%), 114 (19.6\%), 105 (18.1\%), and $23(4.0 \%)$ TIL $200 \mathrm{mg}$ achieved PASI 100, PASI 90-99, PASI 75-89, PASI 50-74, and PASI <50, respectively. On average, PASI 100 patients were younger, lighter, and had shorter disease duration at baseline compared to other response groups. For TIL $100 \mathrm{mg}, \%$ PASI improvement was highest for PASI 100 and least for PASI $<50$ patients for all visits up to week 28 (week 4: 53\%, 46\%, 38\%, 30\%, and $16 \%$; week 28 : $100 \%, 95 \%, 83 \%, 64 \%$, and $33 \%$ for PASI 100, PASI $90-99$, PASI 75 89, PASI 50-74, and PASI $<50$ categories, respectively). Among patients achieving PASI $>50$ at week 28 and continued up to 52 weeks, $\%$ PASI improvement remained consistent or improved from week 28 to week 52 . Similar results were observed for TIL $200 \mathrm{mg}$ as well as subgroup analysis with bio-naive and bioexperienced patients, respectively.

Conclusions: The majority of TIL 100 and $200 \mathrm{mg}$ patients achieved PASI>50 response at week 28 , and PASI improvement was maintained from week 28 to week 52. Among patients achieving $\geq$ PASI 90 at week 28, TIL 100 and $200 \mathrm{mg}$ were associated with rapid improvement by week 4 .

Disclosure of Interest: A. Blauvelt Grant/research support from: AbbVie, Amgen, Boehringer Ingelheim, Celgene, Dermira, Genentech, GSK, Janssen, Lilly, Merck and Co, Novartis, Pfizer, Regeneron, Sandoz, Sanofi Genzyme, Sun UCB, and Valeant, Consultant for: AbbVie, Amgen, Boehringer Ingelheim, Celgene, Dermira, Genentech, GSK, Janssen, Lilly, Merck and Co, Novartis, Pfizer, Regeneron, Sandoz, Sanofi Genzyme, Sun, UCB, and Valeant, Speakers bureau: Eli Lilly, H. Sofen Grant/research support from: Boehringer-Ingelheim, Novartis Pfizer, Janssen, Lilly, Amgen, and Merck and Co., Inc., Consultant for: Novartis, Janssen, and Eli Lilly, Speakers bureau: Novartis, Janssen, and Eli Lilly, K. Papp Grant/research support from: Amgen, Anacor, AbbVie, Active Biotech, Allergan, Astellas, AstraZeneca, Basilea, Bayer, Biogen-ldec, BMS, Boehringer-Ingelheim CanFite, Celgene, Dermira, Eli-Lilly, Forward Pharma, Genentech, GlaxoSmithK line, Janssen, Kyowa Hako Kirin, Kythera, Leo Pharma, Merck and Co, MerckSerono, Novartis, Pfizer, Regeneron, Rigel, Roche, Sanofi-Genzyme, Takeda, UCB, Valeant, Xenon, and Xoma., Consultant for: Amgen, Anacor, AbbVie, Active Biotech, Allergan, Astellas, AstraZeneca, Basilea, Bayer, Biogen-Idec, BMS Boehringer-Ingelheim, CanFite, Celgene, Dermira, Eli-Lilly, Forward Pharma, Genentech, GlaxoSmithKline, Janssen, Kyowa Hako Kirin, Kythera, Leo Pharma Merck and Co, Merck-Serono, Novartis, Pfizer, Regeneron, Rigel, Roche, SanofiGenzyme, Takeda, UCB, Valeant, Xenon, and Xoma., Speakers bureau: Amgen Anacor, AbbVie, Active Biotech, Allergan, Astellas, AstraZeneca, Basilea, Bayer Biogen-Idec, BMS, Boehringer-Ingelheim, CanFite, Celgene, Dermira, Eli-Lilly, 
Forward Pharma, Genentech, GlaxoSmithKline, Janssen, Kyowa Hako Kirin, Kythera, Leo Pharma, Merck and Co, Merck-Serono, Novartis, Pfizer, Regeneron, Rigel, Roche, Sanofi-Genzyme, Takeda, UCB, Valeant, Xenon, and Xoma., M. Gooderham Grant/research support from: AbbVie, Amgen, Boehringer Ingelheim, Celgene, Coherus, Dermira, Galderma, GSK, Jansen, Leo Pharma, Lilly Medimmune, Merck Serono, Novartis, Regeneron, Roche, Sanofi Genzyme, Takeda, UCB, Pfizer, and Valeant., Consultant for: AbbVie, Amgen, Boehringer Ingelheim, Celgene, Coherus, Dermira, Galderma, GSK, Jansen, Leo Pharma, Lilly Medimmune, Merck Serono, Novartis, Regeneron, Roche, Sanofi Genzyme, Takeda, UCB, Pfizer, and Valeant., Speakers bureau: AbbVie, Amgen, Boehringer Ingelheim, Celgene, Coherus, Dermira, Galderma, GSK, Jansen, Leo Pharma, Lilly Medimmune, Merck Serono, Novartis, Regeneron, Roche, Sanofi Genzyme, Takeda, UCB, Pfizer, and Valeant., Y. Zhao Employee of: Sun Pharmaceuticals, S. Lowry Employee of: Sun Pharmaceuticals, A. Mendelsohn Employee of: Sun Pharmaceuticals, J. Parno Employee of: Sun Pharmaceuticals, Q. Li Employee of: Merck and Co., Inc., C. Rosa Employee of: Merck and Co., Inc., K. Reich Grant/research support from: Abbvie, Amgen, Biogen-Idec, Celgene, Centocor, Covagen, Forward Pharma, GlaxoSmithKline, Janssen-Cilag, Leo, Lilly, Medac, Merck and Co, Novartis, Pfizer, Vertex, and Takeda., Consultant for: Abbvie, Amgen, Biogen-Idec, Celgene, Centocor, Covagen, Forward Pharma, GlaxoSmithKline, Janssen-Cilag, Leo, Lilly, Medac, Merck and Co, Novartis, Pfizer, Vertex, and Takeda., Speakers bureau: Abbvie, Amgen, Biogen-Idec, Celgene, Centocor, Covagen, Forward Pharma, GlaxoSmithKline, Janssen-Cilag, Leo, Lilly, Medac, Merck and Co, Novartis, Pfizer, Vertex, and Takeda.

DOI: 10.1136/annrheumdis-2018-eular.2983

\section{AB0956 LEUCINE-RICH ALPHA-2 GLYCOPROTEINIS A PREDICTABLE BIOMARKER FOR THERAPEUTIC RESPONSE AND CLINICAL RELAPSE TO BIOLOGICS, BUT NOT TO APREMILAST IN PATIENTS WITH PSORIASIS}

Y. Shibata ${ }^{1}$, S. Serada ${ }^{2}$, M. Fujimoto ${ }^{2}$, H. Nakajima ${ }^{1}$, S. Sano ${ }^{1}$, T. Naka ${ }^{2,3}$. ${ }^{1}$ Dermatology; ${ }^{2}$ Center for Intractable Immune Disease, Kochi Medical School, Kochi; ${ }^{3}$ Laboratory of Immune Signal, National Institutes of Biomedical Innovation, Health and Nutrition, Osaka, Japan

Background: Leucine-rich alpha-2 glycoprotein (LRG) is a $50 \mathrm{kDa}$ protein produced by hepatocytes, endothelial cells, neutrophils and macrophages, and it was identified as an inflammatory biomarker that correlates with the disease activity of autoimmune diseases such as inflammatory bowel disease and rheumatoid arthritis. We recently reported that LRG could also be a biomarker of psoriasis, which correlated with the clinical severity scores such as Psoriais Area and Severity Index (PASI), Disease Activity Score 28 (DAS-28) and Bath Ankylosing Spondylitis Disease Activity Index (BASDAI) more closely than common inflammation markers like C-reactive protein (CRP) and erythrocyte sedimentation rate (ESR). The biologics are innovative therapies for psoriasis, however they can cause side effects and increase medical cost. Recently, apremilast, a small molecure inhibitor of phosphodiesterase 4 , was approved for treatment of psoriasis in Japan, yet the precise mechanism remains unknown.

Objectives: To explore the eligibility of serum LRG for a biomarker to monitor the responses to biologics and apremilast in psoriasis.

Methods: Antibodies to TNF-alpha, IL-12/IL-23p40, IL-17A and IL-17 receptor A and apremilast were administered in patients with psoriasis vulgaris and psoriatic arthritis ( $n=15,6,14,4$ and 8 respectively). Serum LRG levels were measured by enzyme-linked immunosorbent assay. Serum CRP and ESR, and PASI, DAS-28 and BASDAI were also recorded.

Results: Serum LRG decreased along with the improvement of clinical scores after the administration of biologics, and reflected the change of scores more accurately than CRP and ESR. Furthermore, the LRG levels preceded the changes of clinical symptoms and predicted both primary and secondary treatment failure at the early time point, allowing us to determine if we should increase the doses, discontinue or switch to another drug. In some patients with PASI clear, complete regression of eruption, after biologics, serum LRG rerose from the baseline while their PASI scores remained stable; however, they later relapsed. On the other hand, LRG did not reflect the therapeutic effectiveness of apremilast.

Conclusions: Serum LRG in psoriasis patients would be a sensitive biomarker for predicting the effectiveness and treatment failure of biologics, but not of apremilast. Monitoring LRG levels may enable us to decide the timing of bio-attenuation and to detect the relapse after discontinuation of biologics.

\section{REFERENCE:}

[1] Leucine-rich $\alpha-2$ glycoprotein is an innovative biomarker for psoriasis. J Dermatol Sci. 2017 May;86(2):170-174

Disclosure of Interest: Y. Shibata Grant/research support from: AbbVie GK, S. Serada: None declared, M. Fujimoto: None declared, H. Nakajima Grant/research support from: AbbVie GK, S. Sano Grant/research support from: AbbVie GK, T. Naka: None declared

DOI: 10.1136/annrheumdis-2018-eular.1660

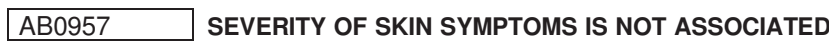 WITH MUSCULOSKELETAL MANIFESTATIONS IN PATIENTS WITH PSORIASIS}

Y. Yamada ${ }^{1,2}$, K. Inui ${ }^{1}$, T. Okano ${ }^{1}$, Y. Sugioka ${ }^{3}$, H. Yoshimura ${ }^{4}$, K. Mamoto $^{1}$ T. Koike ${ }^{5}$, M. Tada ${ }^{6}$, C. Tateishi ${ }^{7}$, E. Yonezawa ${ }^{7}$, A. Natsumi ${ }^{7}$, Y. Fukunaga ${ }^{7}$, D. Tsuruta ${ }^{7}$, H. Nakamura ${ }^{1} .{ }^{1}$ Department of orthopedics surgery, OSAKA CITY UNIVERSITY; ${ }^{2}$ Department of orthopedics surgery, Yodogawa Christian Hospital; ${ }^{3}$ Center for Senile Degenerative Disorders (CSDD), OSAKA CITY UNIVERSITY, Osaka City; ${ }^{4}$ Shirahama Foundation for Health and Welfare; ${ }^{5}$ Search Institute for Bone and Arthritis Disease (SINBAD), Shirahama Foundation for Health and Welfare, Wakayama; ${ }^{6}$ Department of orthopedics surgery, Osaka City General Hospital; ${ }^{7}$ Department of Dermatology, OSAKA CITY UNIVERSITY, Osaka City, Japan

Background: Among patients with psoriasis, risk factors for developing musculoskeletal manifestations, known as psoriatic arthritis (PsA), are not recognised well.

Objectives: The aim of this study is to clarify the relationship between severity of skin disease and arthritis.

Methods: Psoriasis patients referred from dermatologists for assessment of musculoskeletal manifestations between June 2015 and July 2017 were enrolled. Their age, comorbidity, disease duration and treatment were collected. Presence of inflammatory back pain, sacroiliac joint tenderness or enthesitis were examined. Severity of skin symptoms were evaluated by dermatologists in Psoriasis area and severity index (PASI). Psoriatic arthritis screening and evaluation (PASE) and disease activity score (DAS28 ESR) were also evaluated. PsA was diagnosed by The Classification for Psoriatic Arthritis (CASPAR) criteria assisted with musculoskeletal ultrasound examination.

Results: Among 107 patients with psoriasis referred from dermatologists during designated period, 63 patients were diagnosed as PsA. These PsA patients were compared with 44 patients who had no arthritis ( $\mathrm{PsO}$ ). Multiple logistic regression analysis showed neither of age, sex, PASI, disease duration, rheumatoid factor (RF), CRP or Matrix Metalloproteinase-3 (MMP3) had no association with presence of PsA (table 1). Among 63 patients with PsA, those using NSAIDs $(p=0.028)$, those with inflammatory back pain $(p=0.002)$ and male patients $(\mathrm{p}=0.017)$ had significantly high PASI. PASI significantly correlated with age (Spearman's correlation coefficient $R=-0.303$ : $p=0.016)$, body height $(R=0.301$ : $\mathrm{p}=0.019)$ and weight $(\mathrm{R}=0.383: \mathrm{p}=0.002)$, but not with DAS28 $E S R, M M P 3$ or disease duration (table 2 ).

Table 1 multiple logistic regression analysis for presence of PsA

\begin{tabular}{lccc}
\hline variables & OR & $95 \% \mathrm{Cl}$ & $\begin{array}{c}\mathrm{P} \\
\text { value }\end{array}$ \\
\hline age & 0.99 & $0.95-1.03$ & 0.538 \\
sex & 2.13 & $0.66-6.90$ & 0.207 \\
PASI & 0.96 & $0.89-1.03$ & 0.258 \\
disease & 0.99 & $0.96-1.04$ & 0.847 \\
duration & & $0.99-1.01$ & 0.408 \\
RF & 0.99 & $0.76-$ & 0.125 \\
CRP & 2.76 & 10.10 & 0.271 \\
& & $1.00-1.01$ & \\
MMP3 & 1 &
\end{tabular}

Abstract AB0957 - Table 2. Correlations with PASI in patients with PSA (Spearman's correlation)

\begin{tabular}{lcc}
\hline variables & $\mathrm{R}$ & $\begin{array}{c}\mathrm{P} \\
\text { value }\end{array}$ \\
\hline age & -0.303 & 0.016 \\
body height & 0.301 & 0.019 \\
body weight & 0.383 & 0.002 \\
disease & 0.134 & 0.311 \\
duration & & \\
PASE & 0.088 & 0.504 \\
DAS28 ESR & -0.268 & 0.110 \\
RF & 0.152 & 0.273 \\
CRP & 0.211 & 0.105 \\
MMP3 & 0.133 & 0.310 \\
\hline
\end{tabular}

Conclusions: PASI did not associate with presence of arthritis. Furthermore, even DAS28 ESR, reflecting musculoskeletal manifestations, or disease duration did not correlated with PASI among patients with PsA. These indicates severity of skin symptoms is not associated with musculoskeletal manifestations in patients with psoriasis.

Disclosure of Interest: None declared

DOI: 10.1136/annrheumdis-2018-eular.3516 